\title{
OUTROS... LUGARES E MODOS DE "OCUPAÇÃO” DA EDUCAÇÃO FÍSICA NA SAÚDE COLETIVA/SAÚDE PÚBLICA'
}

\author{
Fabio Fortunato Brasil de Carvalho \\ Escola Nacional de Saúde Pública Sergio Arouca - Fiocruz, Manguinhos, Rio de Janeiro, Brasil \\ Yara Maria de Carvalho \\ Universidade de São Paulo, São Paulo, São Paulo, Brasil
}

\begin{abstract}
Resumo
Trata-se de um ensaio de caráter reflexivo a respeito dos lugares da Educação Física na Saúde Coletiva/Saúde Pública, com o intuito de problematizar, e dar visibilidade a, diferentes modos de "ocupação" do campo acadêmico e dos serviços de saúde, especialmente com as práticas corporais. Nesse sentido, demarcaremos alguns cenários a fim de situar a Educação Física e seus movimentos e processos de "ocupação", inspirados pelo número temático dedicado ao "lugar da educação física na saúde pública" da revista Ciência \& Saúde Coletiva. Assim, as práticas corporais como práticas de saúde estão cada vez mais presentes nos serviços de saúde e têm coproduzido redes de cuidado e atenção à saúde, que buscam benefícios para além daqueles que são possíveis observar ou medir e quantificar.
\end{abstract}

Palavras-chave: Práticas Corporais. Saúde coletiva. Atividade Física. Educação Física.

\section{OTHERS... PLACES AND MODES OF 'OCCUPATION' OF PHYSICAL EDUCA- TION IN COLLECTIVE HEALTH / PUBLIC HEALTH}

\begin{abstract}
This is a reflexive essay about the places of Physical Education in Collective Health / Public Health in order to problematize and give visibility to different modes of "occupation" of the academic field and health services, especially with corporal practices. In this sense, we will demarcate some scenarios in order to locate Physical Education and its movements and processes of "occupation", inspired by the thematic number dedicated to the "place of physical education in public health" of the Science and Collective Health. Thus, body practices such as health practices are increasingly present in health services and have coproduced health care and attention networks that seek benefits beyond those that are possible to observe or measure and quantify.

Keywords: Corporal Practices. Public Health. Motor Activity. Physical Education.

\section{OTROS... LUGARES Y MODOS DE 'OCUPACIÓN' DE LA EDUCACIÓN FÍSICA EN LA SALUD COLECTIVA / SALUD PÚBLICA}

\section{Resumen}

Ensayo de carácter reflexivo acerca de los lugares de la Educación Física en la Salud Colectiva / Salud Pública con el propósito de problematizar y dar visibilidad a diferentes

\footnotetext{
${ }^{1} \mathrm{O}$ presente trabalho não contou com apoio financeiro de nenhuma natureza para a sua realização.
} 
modos de "ocupación" del campo académico y de los servicios de salud, especialmente con las prácticas corporales. En ese sentido, demarcaremos algunos escenarios a fin de situar la Educación Física y sus movimientos y procesos de "ocupación", inspirados por el número temático dedicado al 'lugar de la educación física en la salud pública'. Así, las prácticas corporales como prácticas de salud están cada vez más presentes en los servicios de salud y ha co-producido redes de cuidado y atención a la salud, que buscan beneficios más allá de aquellos que son posibles observar o medir y cuantificar.

Palabras clave: Prácticas Corporales. Salud Pública. Actividad Motora. Educación Física.

\section{Introdução}

"O lugar da educação física na saúde pública", tema norteador da edição de abril de 2016 da revista Ciência \& Saúde Coletiva (C\&SC), da Associação Brasileira de Saúde Coletiva (ABRASCO), inspirou a discussão que propomos aqui, haja vista nossas trajetórias de pesquisa, formação e intervenção com as práticas corporais contemporâneas no campo da Saúde Coletiva/Saúde Pública. As práticas corporais como práticas de saúde têm, gradativa e insistentemente, se espalhado nos espaços e territórios dos serviços de saúde e entornos com o trabalho das equipes de saúde e têm coproduzido redes de cuidado e atenção à saúde.

O presente ensaio destaca outros... lugares e modos de "ocupação" da Educação Física (EF), com o intuito de chamar a atenção para essa figura conceitual crítica, "práticas corporais", à medida que ela faz um contraponto à discussão centrada na atividade física (AF) e, ao mesmo tempo, amplia o espectro de observação mobilizado por essas práticas. Como ações de saúde, as práticas corporais são tecnologias de cuidado porque abrem possibilidades para encontros e experimentações no que se refere ao cuidado e à atenção à saúde.

$\mathrm{Na}$ edição da $C \& S C$ citada, foram publicados artigos que responderam à provocação temática fundamentados pela perspectiva epidemiológica. Aqui, enfatizaremos outras perspectivas teórico-conceituais, orientadas pelas ciências sociais e humanas (CS\&H), com o propósito de dar visibilidade a outros modos de "ocupação" da EF e, assim, ampliar as possibilidades para pensar e propor estratégias e ações voltadas para o cuidado e a atenção em saúde.

\section{Notas breves sobre a relação entre Educação Física e epidemiologia}

A edição da $C S \& C$ nos remete, segundo Minayo e Gualhano (2016), aos documentos da Organização Mundial da Saúde (OMS) e às pesquisas nacionais sobre inatividade física e sedentarismo como fatores de risco para as doenças crônicas não transmissíveis (DCNTs) e propõe a AF como fator de proteção à saúde. De fato, há evidencias a respeito das correlações entre AF e enfermidades, amplamente difundidas na literatura e na mídia impressa e televisiva. Martinez et al. (2013) afirmam que as ações de promoção da saúde, no contexto brasileiro, em especial as práticas corporais e a AF, foram interpretadas como resposta à "epidemia" de DCNTs.

Sobre o lugar da EF na Saúde Coletiva/Saúde Pública inicialmente apontado, Minayo e Gualhano (2016), ao apresentarem a edição de abril, destacam as disparidades de duas grandes pesquisas nacionais, realizadas pelo Instituto Brasileiro de Geografia e Estatística (IBGE) e pelo Ministério do Esporte, que se propuseram a medir o sedentarismo entre os brasileiros, nas quais respectivamente encontraram que 80\% (em 2010) e 45,9\% (em 2016) dos brasileiros e brasileiras eram sedentários. As autoras afirmam que essa disparidade se dá possivelmente por causa das metodologias usadas, o que foi apontado por Palma e Vilaça (2010), ao abordarem os limites teórico-metodológicos dos estudos epidemiológicos com a mesma proposição, ou de medir a inatividade física. Os autores afirmam haver imprecisão proporcio- 
nada por incongruências entre os valores das distintas medidas de sedentarismo ou inatividade física em diferentes estudos.

Nogueira e Bosi (2017) afirmam que, ao naturalizar a linguagem do risco do sedentarismo, a EF teve a epidemiologia como o primeiro núcleo de vinculação da interface com a Saúde Coletiva/Saúde Pública. Afirmam também que ocorreram reflexões vindas das CS\&H, que ampliaram a visão da interface entre elas, a partir do reconhecimento da natureza multidimensional da cultura corporal. Para Martinez et al. (2013), desde os anos 80 do século passado a EF incorporou influências das CS\&H, contudo, a ênfase foi nas questões educacionais, com a saúde não sendo privilegiada.

Relendo a bibliografia a respeito dessa problemática, Castiel, Guilam e Ferreira (2010) alertam para a necessidade de uma análise crítica da epidemiologia no que se refere aos fatores de risco, sob pena de criação de uma confiança desmedida em torno de sua capacidade de responder aos complexos problemas de saúde pública. Para os autores, esse saber é necessário para as análises e intervenções sanitárias, mas está longe de ser suficiente, como, por vezes, parece se autorizar a assumir tal papel.

Fatores de risco, para Minayo (2008), são a expressão do modo de vida de grupos populacionais, sendo imperativo abrir a ciência epidemiológica à investigação dos aspectos simbólicos, tais como valor e significado, do risco e seus determinantes. Afirma a autora que vivências desiguais constroem formas diversas de pensar, sentir e agir peculiares a respeito da saúde e da doença, não havendo saúde nem construção social da saúde em abstrato e fora da sociedade que as gera.

Sob outra perspectiva, Potvin e Chabot (2014) destacam que a epidemiologia (clássica) provou sua força de compreensão sobre como os comportamentos se transformam em fatores de risco relacionados a doenças e assim se tornou a metodologia preferencial para pesquisas em saúde. Contudo, ponderam que as necessidades de saúde não são definidas de forma normativa, que elas são socialmente estabelecidas por meio de pactos entre sujeitos de contextos culturais, sociais e econômicos, envolvendo acesso a vários serviços (CARVALHO et al., 2014), dentre eles os serviços de atenção à saúde.

Minayo e Gualhano (2016) ressaltam que as discussões sobre AF estão intimamente vinculadas aos significados do corpo na atualidade. Para elas, a saúde e a qualidade de vida não devem ser vistas de forma mecânica ou isolada do contexto contemporâneo, pois o corpo biológico é também uma construção simbólica saturada de sentidos. Contudo, há destaque, na edição temática do periódico, para os benefícios biológicos e orgânicos da AF como melhora da postura, tornar os músculos mais eficientes, ajudar a diminuir o excesso de peso e o acúmulo de gordura, melhorar o sistema imunológico e auxiliar a reduzir os efeitos de DCNTs e alterações nas taxas de colesterol (MINAYO; GUALHANO, 2016).

Mássimo e Freitas (2014), ao pesquisar riscos para DCNTs na ótica de participantes do Sistema de Vigilância de Fatores de Risco e Proteção para DCNT (VIGITEL), baseadas nas reflexões dos grupos participantes de sua pesquisa, escrevem sobre a existência de representações centradas na impossibilidade de considerar a saúde em primeiro plano e constantemente na vida cotidiana, devido à existência do desejo de viver prazeres e satisfações que, muitas vezes, não condizem com a "determinação" do que é ser saudável. E lembram que há a necessidade de transgressões para as pessoas sentirem-se vivas e mais fortes que a morte.

Para os objetivos deste ensaio, é importante reconhecer que a adesão a modos de vida saudáveis é uma construção social dependente, dentre outros fatores, das representações sobre risco em saúde, já que os sujeitos acumulam uma bagagem psicossocial pelas vivências e interações que se renovam e se reconstroem, além do desejo de viver prazeres e aventuras e da impossibilidade de pensar em saúde e realizar o modelo prescritivo das ações de saúde o tempo todo (MÁSSIMO; FREITAS, 2014). Assim, afirmamos que as práticas corporais são parte dessa construção social. 
Destaca-se ainda, nessa breve análise sobre a relação entre a epidemiologia e a EF, que novas evidências têm questionado o saber vigente relacionado, por exemplo, às recomendações de duração e intensidade de AF para a saúde pública a partir de um erro na tradução do conhecimento (WARBURTON; BREDIN, 2016; 2017), mostrando a importância da problematização apresentada, de forma sucinta, neste tópico.

Ou, ainda, outras interpretações, conforme apontaram Silva et al. (2017), ao afirmarem que a AF é um comportamento multidimensional que reflete os modos de vida das sociedades, e sua interface com a saúde ultrapassa o gasto energético e a prevenção de doenças, sendo necessária uma abordagem das recomendações vigentes com base em uma compreensão mais ampla da AF, o que demandaria outras perspectivas de pesquisa e oferta dessas práticas em âmbito populacional. Recente relatório do Programa das Nações Unidas para o Desenvolvimento (PNUD) também abordou tais possibilidades (PNUD, 2017).

\section{Os cenários nos serviços públicos de saúde}

Cândido, Palma e Assis (2016) afirmam que o atual apelo para a prática de AF tem colaborado para uma ideia biologicista de intervenção, ou uma "bio-educação-física" na perspectiva de Mendes e Carvalho (2016), ao valorizar a figura dos "especialistas da saúde", dos "conselheiros privados", reproduzindo uma "biopolítica informacional" em detrimento do cuidado em saúde (CARVALHO; GOMES; FRAGA, 2015).

No "plano macro" das políticas, houve a inclusão da AF na Lei Orgânica da Saúde, através da Lei $n^{\circ} 12.864 / 2013$. No "plano micro", quando observamos os cenários ${ }^{2}$ dos serviços públicos de saúde, especialmente as Unidades Básicas de Saúde e os Centros de Apoio Psicossocial, é evidente o aumento de iniciativas e programas cujo foco é a AF e as práticas corporais no contexto do Sistema Único de Saúde (SUS), em especial na Atenção Básica (AB)/Atenção Primária em Saúde (APS). São cada vez mais frequentes os depoimentos dos profissionais de saúde qualificando esse tipo de iniciativa (CARVALHO; FERREIRA NETO, 2016; CARVALHO; MANOEL, 2015): quatro em cada dez UBS desenvolvem iniciativas com atividade física, mais frequentemente grupos de caminhada (GOMES et al., 2014).

Foram várias ações que contribuíram para o incremento dessas práticas no SUS. Em 2003, a OMS publica a Estratégia Global sobre Alimentação Saudável, Atividade Física e Saúde e, ainda que seu foco tenha sido as DCNTs (CRUZ, 2010), foi uma iniciativa que demarcou a importância do tema para a saúde das populações. Em 2006, o Ministério da Saúde (MS) publicou a Política Nacional de Promoção da Saúde (PNPS), primeiro texto oficial em que aparece a expressão atividade física/práticas corporais. E entre 2005 e 2010, houve um incentivo técnico e financeiro dado pelo MS do Brasil para fortalecer a PNPS, com a maior parte das ações sendo relacionada a tais práticas.

Mais recentemente, em 2011, foi lançado o Plano de Ações Estratégicas para o Enfrentamento das DCNTs no Brasil (BRASIL, 2011), no qual as práticas corporais e AF (expressão cunhada com a barra dividindo-as) são enunciadas como ações de Promoção da Saúde, o que foi debatido por Carvalho e Nogueira (2016). Nesse mesmo ano, foi criado o Programa Academia da Saúde. Além dele, há relatos na literatura evidenciando que as equipes da Estratégia de Saúde da Família (ESF) e do Núcleo de Apoio à Saúde da Família (NASF), ${ }^{3}$ conforme denominado à época, também ofertam ações relacionadas à $\mathrm{AF}$ e às práticas corpo-

\footnotetext{
${ }^{2}$ Por cenários, referimo-nos a conjuntos de elementos presentes na realidade cotidiana (na qual os serviços de saúde estão inseridos) e que conformam as ofertas de cuidado, dentre elas as práticas corporais. Assim como também elementos mais amplos presentes na pesquisa, ensino, entre outros, ou seja, panoramas e não só a ideia de lugar físico.

3 Atualmente, através da atual Política Nacional de Atenção Básica - Portaria GM/MS n 2.436, de 21 de setembro de 2017, passou a denominar-se Núcleo Ampliado de Saúde da Família e Atenção Básica (NASF-AB). 
rais, dentre outras (CARVALHO; NOGUEIRA, 2016; FLORINDO et al., 2016). Em 2014, a PNPS foi revisada, e as práticas corporais e a AF foram mantidas como ações prioritárias.

Mas é importante mencionar que em 2006, com a publicação da PNPS, aparece na cena uma nova questão, de natureza conceitual: a expressão atividade física/práticas corporais. Essa dupla noção irá desencadear junto aos grupos de pesquisa, nos cursos de formação em saúde, mas também nos diferentes cenários da saúde, novas composições para pensar o cuidado e as práticas de saúde.

Desde o início da década de 1980, a área da EF já apontava alguns questionamentos a respeito do viés biologicista e higienista (CASTELLANI, 1988; SOARES, 1990). Outro exemplo pode ser a provocação de Carvalho (1995) com a ideia de "mito", mais precisamente com o livro $O$ 'mito' da atividade física e saúde, no qual a autora afirma que a saúde pública, a mídia impressa e televisiva, as indústrias farmacêuticas e as de cosméticos vendem a noção de saúde, transformando-a em mercadoria.

Reforça-se a ideia de que o indivíduo é o único responsável pela sua saúde e pela sua qualidade de vida, fundamentada no pressuposto de que as doenças são causadas devido à negligência do indivíduo em relação ao seu corpo, caracterizando uma abordagem culpabilizante. Destaca-se ainda que remonta do final do século passado, mais especificamente entre 1998 e 1999, a organização de grupos de pesquisa que utilizaram a Saúde Coletiva como referencial para investigar e questionar a relação entre AF e saúde (MARTINEZ et al., 2013).

Mendes e Carvalho (2016) escreveram sobre a bio-Educação Física e as práticas corporais como dois percursos que aproximam EF e Saúde Coletiva/Saúde Pública, perspectivas distintas que dialogam com o campo: a primeira, pelo caminho da epidemiologia e a outra, pelo das ciências humanas e sociais. São propostas diferentes, não excludentes, e aparentemente há consenso de que ambas são importantes para pensar as dimensões do cuidado à saúde, do corpo, da saúde e dos modos singulares de andar a vida (CARVALHO; FERREIRA NETO, 2016).

Martinez et al. (2013), em complemento, identificaram duas perspectivas teóricometodológicas antagônicas na relação entre EF e Saúde Pública: o paradigma dos "fatores de risco", no qual são priorizadas ações de prevenção, controle e tratamento de doenças, e, outra, iniciativas de superação dessa perspectiva, a partir do que os autores denominaram de paradigma promocional, baseado na concepção ampliada de saúde. Assim, para os autores, o reconhecimento da relevância do SUS, política e socialmente, na EF foi um grande avanço, mas há distintas matizes analíticas e práticas em disputa nesse processo.

O conceito de práticas corporais não aparece na edição mencionada da revista, ainda que o debate no campo da Saúde Coletiva/Saúde Pública, e especialmente no da Promoção da Saúde, agregue as diferenças e as especificidades a que remetem essas distintas expressões (CARVALHO, 2006; CARVALHO, 2010; LAZZAROTTI FILHO et al., 2010; FRAGA; CARVALHO; GOMES, 2013; DAMICO; KNUTH; 2014; CABALLERO, 2015; CARVALHO, 2016; CARVALHO; FERREIRA NETO, 2016), o que denota uma tensão existente, no campo acadêmico e nas intervenções, entre diferentes teorias sociais que sustentam a correlação de forças entre o discurso hegemônico amparado pelo conceito restrito de AF e as críticas e possibilidades de superação propostas pelo termo "práticas corporais" no campo da Saúde Pública (MARTINEZ et al., 2013).

A expressão "prática corporal" é uma figura conceitual crítica, que faz um contraponto à discussão centrada na AF. É uma noção que carrega consigo uma proposição para além da intervenção em saúde, à medida que instiga uma reflexão a respeito das práticas de saúde como tecnologias de cuidado (CARVALHO, 2010). O propósito é abrir possibilidades para encontros e novas experimentações no que se refere ao corpo e aos seus cuidados. É assumir a complexidade a que remete a noção "cuidado" que, em última instância, nos exige 
retomar a própria noção "vida", a favor dela. Deslocar nosso olhar da dimensão técnica da AF para o viver, com as práticas corporais.

É uma perspectiva que problematiza os modos de existir: o movimentar-se, ou o ser humano em movimento e implica nos atermos aos processos de produção de saúde. Nessa circunstância, o movimento não busca adequação à proposição teórico-conceitual ou pragmática da AF e saúde, mas repensar os melhores movimentos que as experiências vividas trazem ou que, por viver, pedem (CABALLERO, 2015). Para Martinez et al. (2013), as práticas corporais têm o objetivo de superar fins puramente mecânicos e/ou utilitários, buscando dimensões relacionadas à ludicidade, ao prazer, à solidariedade, à criatividade, entre outros.

Outros modos de ocupação da EF na Saúde Coletiva/Saúde Pública podem ser confirmados em artigos e livros publicados especialmente no campo e também em relatos de quem vive o cotidiano dos serviços de saúde. A AB/APS traz exemplos de outros modos de ocupação da EF sobre os quais abordamos neste texto. Caballero (2015), baseado em relatos da IV da Mostra Nacional de Atenção Básica/Saúde da Família, realizada em 2014, afirma que os objetivos contemplados pelas práticas corporais dialogam com necessidades decorrentes de adoecimentos, mas também com demandas dos participantes ou pelos próprios proponentes, quando constatam limitações na adesão às suas iniciativas. $\mathrm{O}$ autor relata, por exemplo, caminhadas pensadas e realizadas para visitação de pessoas da comunidade que estavam acamadas, ou seja, aparentemente buscando unir benefícios biológicos com sociais e comunitários (CABALLERO, 2015).

Já Becalli e Gomes (2014), baseados em pesquisa com usuários de um programa municipal que estimula a prática de $\mathrm{AF}$, afirmam que há outras possibilidades além dessa prática, como a produção de encontros, de compartilhamento do cotidiano, que, em alguns casos, constituem-se como elemento mobilizador dos indivíduos e, sobretudo, como elemento de produção de saúde. Complementam afirmando que os usuários do programa, ao relatarem os benefícios percebidos, destacam a produção de sociabilidades e a sensação de bem-estar. Afirmam ainda que o programa, mais que um espaço voltado exclusivamente à prática de $\mathrm{AF}$, é um espaço de produção de sentidos e de subjetividades.

Já os relatos, disponíveis no sítio eletrônico Comunidade de Práticas (https://novo.atencaobasica.org.br) - um espaço virtual para o compartilhamento de experiências de profissionais, gestores e demais envolvidos no cotidiano dos serviços da AB/APS, com o objetivo de construção de conhecimentos e práticas - apresentam experiências com práticas corporais ofertadas pelas equipes ESF e NASF. Em um deles, segundo a autora, misturam-se atividades que experimentam benefícios biológicos, tais como fortalecimento muscular, melhora do equilíbrio, agilidade e coordenação, com aquelas que objetivam inclusão social, educação em saúde, atividades culturais, rodas de leitura, cursos intersetoriais sobre alimentação saudável, entre outras atividades.

Para ela, tais ações promovem melhora da qualidade de vida por trabalharem o físico, o psíquico e o social, sendo, portanto, uma atividade global. A autora destaca que foi observada mudança também no modo de trabalho dos profissionais, que deixaram as palestras e as informações, a fim de privilegiar a educação em saúde de modo participativo, que busca proporcionar o empoderamento do usuário.

Outro exemplo, relata outra autora, surgiu a partir da procura recorrente de membros da comunidade com DCNTs identificados pela ESF, em especial pelo agente comunitário de Saúde (ACS) da área. Inicialmente a equipe de saúde, incluídos residentes multiprofissionais em Saúde da Família, foi conhecer o território, o perfil da população, possíveis locais onde a atividade poderia acontecer e quais as necessidades de saúde da comunidade. Em seguida, propuseram encontros sobre temas variados.

Durante os encontros, são realizados momentos de Educação em Saúde sobre diversos temas de interesse dos participantes do grupo com atividades de lazer, também com o propó- 
sito de qualificar a convivência. Devido à dificuldade com o espaço, houve a necessidade de contar com o apoio de uma participante do grupo, que cedeu o quintal de sua casa, mais adequado à realização dessas práticas. No primeiro dia da atividade, no horário divulgado, só havia uma participante, ao ligarem o som e anunciar a atividade, nas palavras da autora: "como num passe de mágica, as portas das casas começaram a se abrir e grupos de pessoas juntaram-se e aumentou o número de participantes". Ao final, a atividade contava com a presença de crianças, adultos e idosos. Destaca-se ainda a articulação interprofissional, através da equipe do NASF, entre a equipe de residentes de Saúde da Família e os acadêmicos.

A inexistência de equipamentos sociais "saudáveis", como praças, quadras ou associação comunitária, foi uma questão trabalhada nesse grupo, por meio de rodas de conversa, utilizadas como método avaliativo das ações do grupo, nas quais buscaram-se alternativas como a articulação de outros setores para a busca de apoio e a discussão das necessidades de saúde do território. A autora conclui que os grupos de práticas corporais na AB/APS são experiências que possibilitam a vivência de um fazer cotidiano pautado na perspectiva do cuidado em saúde, para além do físico, com base na clínica ampliada, sendo estes espaços de convivência, lazer, troca de experiências e saberes em saúde.

Há ainda o relato, na atenção especializada ambulatorial, por parte de Martinez et al. (2013), segundo o qual, mesmo com a necessidade do saber específico de determinadas patologias e condições de saúde, buscou-se abranger não apenas o viés biológico dos corpos, intencionando retirar o foco da doença e da AF como única opção de solução ou de atenuação de sintomas e ampliar a autonomia dos sujeitos mediante maior conhecimento interno e externo do corpo. Afirmam ainda ser possível criar vínculos, assim como propor e desenvolver novas práticas corporais (MARTINEZ et al., 2013), ainda que não seja uma característica inerente a esse lócus da atenção à saúde.

Assim, são exemplos de outros modos de ocupação possíveis que propiciam encontros e experimentações que refletem, também, o cuidado e a atenção à saúde. Dada a opção da edição da $C S \& C$ de privilegiar a perspectiva epidemiológica, é compreensível que questões não sejam contempladas, como as mudanças no modo de trabalho dos profissionais buscando o empoderamento do usuário, a partir de modos participativos de educação em saúde, ou ainda a inexistência de equipamentos sociais "saudáveis", o que gerou mobilização individual e da comunidade, tanto na disponibilização do espaço como na busca de alternativas, e a articulação interprofissional e interdisciplinaridade. Contudo, afirmamos que elas estão diretamente relacionadas às possibilidades da realização das práticas corporais por sujeitos e coletividades e, consequentemente, aos benefícios que elas proporcionam, não só os biológicos.

\section{Considerações}

Buscou-se, através da compreensão das práticas corporais como práticas de cuidado e saúde, apresentar outros... lugares e modos de "ocupação" da EF na Saúde Coletiva/Saúde Pública. Lugares e modos que consideram desejos e possibilidades, tais como aquelas que estão presentes no cotidiano dos serviços de saúde, seja o espaço da prática, o da mobilização da comunidade, o do empoderamento dos usuários, o das estratégias para ampliar a participação, o da inclusão social, o do fortalecimento de laços sociais através da solidariedade, o das atividades culturais que têm interseção com as práticas corporais, o dos encontros e compartilhamentos dos momentos de vida, entre outras.

Alguns relatos e algumas referências da literatura confirmam que as práticas corporais como práticas de saúde estão cada vez mais presentes nos serviços de saúde e, assim, têm coproduzido redes de cuidado e atenção à saúde, que buscam benefícios para além daqueles que são possíveis observar ou medir e quantificar. Destacamos a importância da epidemiolo- 
gia na construção do conhecimento e das práticas de Saúde Coletiva/Saúde Pública, assim como a necessidade de ampliarmos o território de busca por novas práticas, a partir de outros referenciais.

Entre discurso, práticas e intencionalidades, existem caminhos, lugares, formas distintas de operacionalizar as interfaces entre EF e Saúde Coletiva/Saúde Pública. Assim, tão importante quanto mensurar e quantificar a relação entre a $\mathrm{AF}$ e os benefícios individuais e coletivos para a saúde, é compreender os sentidos e os significados atribuídos às práticas pelos sujeitos: o que os faz, a partir de sua história e contexto de vida, praticá-las ou não?

As práticas corporais, como práticas de cuidado, ao buscar efetivar as possibilidades para encontros e novas experimentações no que se refere ao corpo e à saúde, ao deslocar o olhar da dimensão técnica da AF para o viver, propicia outros... lugares e modos de "ocupação" da EF na Saúde Coletiva/Saúde Pública que vão além da dimensão pragmática da AF e saúde, já que a literatura acadêmico-científica prova que esta tem correlação com a doença e, ao mesmo tempo, prova a baixa adesão aos programas e iniciativas voltados para esse tipo de prática. Parece-nos que colocar em diálogo diferentes perspectivas poderá, a curto e médio prazo, responder àquela e a tantas outras questões que, há tempos, continuam em aberto.

\section{Referências}

BECCALLI, M. B.; GOMES, I. M. Mais que atividade física: usos e entendimentos da saúde e do serviço de orientação ao exercício da prefeitura municipal de vitória entre usuários do serviço. Revista Brasileira de Ciências do Esporte, Florianópolis, v. 36, n. 2, supl., p. S26S43, abr./jun. 2014

BRASIL. Ministério da Saúde. Secretaria de Vigilância em Saúde. Departamento de Análise de Situação de Saúde. Plano de ações estratégicas para o enfrentamento das doenças crônicas não transmissíveis (DCNT) no Brasil 2011-2022 - Brasília: Ministério da Saúde, 2011. 160 p. (Série B. Textos Básicos de Saúde).

CABALLERO, R. M. S. Pedagogia das vivências corporais: educação em saúde e culturas de corpo e movimento. 2015. 183 p. Tese (Doutorado) - Universidade Federal do Rio Grande do Sul, Faculdade de Educação, Programa de Pós-graduação em Educação, Porto Alegre, 2015 .

CÂNDIDO, C. M.; PALMA, A.; ASSIS, M. R. A representação da Educação Física no quadro medida certa/ 90 dias para reprogramar o corpo exibido pela TV Globo. Rev. Brasileira de Educação Física e Esporte, São Paulo, v. 30, n. 2, p. 345-57, abr./jun. 2016.

CARVALHO, F. F. B. Práticas corporais e atividades físicas na Atenção Básica do Sistema Único de Saúde - ir além da prevenção das doenças crônicas não transmissíveis é necessário. Movimento, v. 22, n. 2, p. 647-658, 2016.

; NOGUEIRA, J. A. D. Práticas corporais e atividades físicas na perspectiva da Promoção da Saúde na Atenção Básica. Ciência \& Saúde Coletiva, v. 21, n. 6, p. 1.829-1.838, 2016.

CARVALHO, Y. M. O “mito" da atividade física e saúde. São Paulo: Editora Hucitec, 1995. 133p. 
Promoção da saúde, práticas corporais e atenção básica. Revista Saúde da Família, Brasília, v. 2, n.7, p.33-45, 2006.

As práticas corporais como práticas de saúde e de cuidado no contexto da promoção da saúde. 2010. 101 p. Tese (Livre-docência)- Faculdade de Saúde Pública, Universidade de São Paulo, São Paulo, 2010.

; GOMES, I. M.; FRAGA, A. B. EF + Ciências Humanas + Saúde. In: STIGGER, M. P. (Org.). EF + Humanas. Campinas: Autores Associados, 2015.

; MANOEL, E. J. A survey of body practices and primary health care in adistrict of São Paulo, Brazil. Motriz: Revista de EF, v. 21, p. 75-83, 2015. Disponível em: http://www.scielo.br/pdf/motriz/v21n1/1980-6574-motriz-21-01-00075.pdf. Acesso em: 27 nov. 2017.

; FERREIRA NETO, J. L. Atividade física e práticas corporais: uma perspectiva micropolítica. In: KASTRUP, V.; MACHADO, A. M. (Org.). Movimentos micropolíticos em saúde, formação e reabilitação. Curitiba: Editora CRV, 2016.

CARVALHO, A.I. et al. Concepções e abordagens na avaliação em promoção da saúde. In: BRASIL. Conselho Nacional de Secretários de Saúde (CONASS). Avaliação em promoção da saúde: uma antologia comentada da parceria entre Brasil e a CACIS da universidade de Montreal de 2002 a 2012. Brasília: CONASS, 2014. p. 38-50.

CASTELLANI FILHO, L. Educação Física no Brasil: a história que não se conta. Campinas, SP: Editora Papirus, 1988.

CASTIEL, L. D.; GUILAM; M. C. R.; FERREIRA; M. S. Correndo o risco: uma introdução aos riscos em saúde. Rio de Janeiro: Editora Fiocruz, 2010.

CRUZ, D. K. A. Da promoção à prevenção: o processo de formulação da política nacional de promoção da saúde no período de 2003 a 2006. 2010. 118 p. Dissertação (Mestrado em Ciências da Saúde) - Centro de Pesquisas Aggeu Magalhães, Fundação Oswaldo Cruz, Recife, 2010.

DAMICO, J. G. S.; KNUTH, A. G. O (des)encontro das práticas corporais e atividade física: hibridizações e borramentos no campo da saúde. Movimento, Porto Alegre, v. 20, n. 1, p. 329-350, 2014.

FLORINDO, A. A. et al. Promoção da atividade física e da alimentação saudável e a saúde da família em municípios com academia da saúde. Revista Brasileira de Educação Física e Esporte, São Paulo, v. 30, n. 4, p. 913-24, out./dez. 2016.

FRAGA, A. B.; CARVALHO, Y. M.; GOMES, I. M. As práticas corporais no campo da saúde. In: FRAGA, A. B; CARVALHO, Y. M.; GOMES, I. M. (Org.). As práticas corporais no campo da saúde. São Paulo: Hucitec, 2013.

GOMES, G. A. O. et al. Characteristics of physical activity programs in the Brazilian primary health care system. Cadernos de Saúde Pública, Rio de Janeiro, v.30, n.10, out. 2014. 
LAZZAROTTI FILHO, A. et al. O termo práticas corporais na literatura científica brasileira e sua repercussão no campo da EF. Movimento, Porto Alegre, v. 16, n. 1, p. 11-29, jan./mar. 2010.

MARTINEZ, J. F. N. et al. Práticas corporais e SUS: tensões teóricas e práticas. In: FRAGA, A. B; CARVALHO, Y. M.; GOMES, I. M. (Org.) As práticas corporais no campo da saúde. São Paulo: Hucitec, 2013. p. 139-77.

MÁSSIMO, E. A. L.; FREITAS, M. I. F. Riscos para doenças crônicas não transmissíveis na ótica de participantes do Vigitel. Saúde e Sociedade, São Paulo, v.23, n.2, p.651-663, 2014.

MENDES, V. M.; CARVALHO, Y. M. Práticas corporais e clínica ampliada. São Paulo/Brasília: Hucitec/CAPES, 2016.

MINAYO, M. C. S. O desafio do conhecimento: pesquisa qualitativa em saúde. 11. ed. São Paulo: Hucitec, 2008. 407 p.

MINAYO, M. C. S; GUALHANO, L. Press release. Ciência \& Saúde Coletiva, v. 21, n.4, p. 998, 2016.

NOGUEIRA, J. A. D.; BOSI, M. L. M. Saúde Coletiva e Educação Física: distanciamentos e interfaces. Ciência \& Saúde Coletiva, v. 22, n.6, p. 1913-1922, 2017.

PALMA, A.; VILAÇA, M. M. O sedentarismo da epidemiologia. Revista Brasileira de Ciências do Esporte, Campinas, v. 31, n. 2, p. 105-119, jan. 2010.

POTVIN, L.; CHABOT, P. O esplendor e a miséria da epidemiologia na avaliação da promoção da saúde. In: BRASIL. Conselho Nacional de Secretários de Saúde (CONASS). Avaliação em promoção da saúde: uma antologia comentada da parceria entre Brasil e a CACIS da universidade de Montreal de 2002 a 2012. Brasília: CONASS, 2014. p. 23-37.

PROGRAMA DAS NAÇÕES UNIDAS PARA O DESENVOLVIMENTO (PNUD). Relatório de Desenvolvimento Humano Nacional - Movimento é Vida: Atividades Físicas e Esportivas para Todas as Pessoas: 2017. Brasília: 2017.

SILVA, K. S. et al. Physical activity as part of daily living: moving beyond quantitative recommendations. Preventive Medicine, v. 96, p. 160-162, mar. 2017.

SOARES, C. L. A influência do pensamento médico higienista na Educação Física no Brasil: 1850-1930. 1990. 247 p. Dissertação (Mestrado em Educação: Filosofia e História da Educação) - Pontifícia Universidade Católica de São Paulo (PUC/SP), São Paulo, 1990.

WARBURTON, D.; BREDIN, S. Reflections on physical activity and health: what should we recommend? Canadian Journal of Cardiology, v. 32, n. 4, p. 495-504, 2016. Disponível em: http://www.onlinecjc.ca/article/S0828-282X(16)00064-7/pdf. Acesso em: 01 dez. 2017.

Health benefits of physical activity: a systematic review of current systematic reviews. Current Opinion in Cardiology, v. 32, n. 5, p. 541-556, set. 2017. 
Recebido em: 02/02/2018

Revisado em: 27/03/2018

Aprovado em: 29/03/2018

Endereço para correspondência:

fabiofbcarvalho@gmail.com

Fabio Fortunato Brasil de Carvalho

Escola Nacional de Saúde Pública Sergio Arouca

Rua Leopoldo Bullhões, 1480 -Manguinhos

Rio de Janeiro. CEP: 21041-210 